\title{
Synthesizing high aspect ratio Aluminum Oxide nanowires from highly-ordered anodic self-assembled templates
}

\author{
Mastooreh Seyedi $^{\mathrm{a}, *}$, Mozhdeh Saba ${ }^{\mathrm{b}}$ \\ ${ }^{a}$ Department of Materials Science and Engineering, Clemson University, 161 Sirrine Hall, Clemson, SC \\ 29634, USA \\ ${ }^{b}$ Department of Polymer Engineering and Color Technology, Amirkabir University of Technology, Tehran, \\ Tehran, Iran
}

\begin{abstract}
Aluminum Oxide and its nanostructures are attracted the attention of researchers due to their special materials properties such as: high electrical insulation, high mechanical strength, corrosion resistance, chemical stability, and low thermal conductivity. Furthermore, Aluminum Oxide nanowires shows large surface area as well as highly electropositive surface, which makes them suitable candidates for water purification technology applications. One of the main challenges, which limited the usage of Aluminum Oxide nanowires, is high cost and complex fabrication methods for Aluminum Oxide synthesizing such as chemical vapor deposition (CVD) techniques. On the other hand, electrochemical methods such as anodizing/etching techniques show high controllability over chemical composition, morphology, and crystalline structure of nanowires. In this research, a room temperature two-steps anodization procedure is developed to fabricate a highly-ordered self-assembled templates. Furthermore, the etching method is used to convert this synthesized self-assembled template into Aluminum Oxide nanowires. The results show that the proposed electrochemical method maintains a highly-ordered morphology as well as industrially acceptable controllability over crystalline structure of nanowires, which could be used to optimize the procedure for industrial applications due to low cost and simple experimental setup.
\end{abstract}

Keywords: Aluminum Oxide nanowires, Highly-ordered Aluminum Oxide, Two-step anodization, Chemical etching, Self-assembled templates

\section{Introduction}

Aluminum Oxide nanowires have exceptional mechanical, electrical, and optical properties (e.g. High dielectric coefficient, semiconductivity, high mechanical strength, photonic crystals, etc.), which make them suitable for micro-electronic device applications [1-21]. Although, synthesizing the Aluminum Oxide nanostructures in large volume for industrial applications, due to their expensive and time consuming production methods such as: chemical

\footnotetext{
*Corresponding author: Mastooreh Seyedi Department of Materials Science and Engineering, Clemson University, 161 Sirrine Hall, Clemson, SC 29634, USA Email: sseyedi@clemson.edu Phone: 1-864-624-2838 
vapor deposition (CVD) techniques or electrophoretic based methods [22-32], limited their application for opteoelectronic devices or water purification technologies. On the other hand, novel fabrication techniques such as electrochemical anodization methods have shown more accurate controllability over materials morphology and chemical composition of nanostructures in comparison to conventional production techniques as well as their easier scalability for industrial applications [33-52]. As a result, the electrochemical anodization techniques have a potential to pave the way for the industrial scale production of nanostructures with a highly controlled morphology as well as their chemical composition and crystalline structure [53]. In this research, two-steps anodization method is combined with chemical etching in order to fabricate highly-ordered Aluminum Oxide nanowires from self-assembled templates. Furthermore, this novel room temperature two-steps anodization technique, which is used to produce a highly-ordered template, as well as its morphological properties will be investigated in details. Finally, morphological properties as well as chemical structure of synthesized Aluminum Oxide nanowires will be studied in order to examine their crystalline structures as well as controllability of nanowires' chemical composition. All the experiments in this research are done at room temperature, which paves the way for its industrial scale generalizations due to technical difficulties related to costly temperature controlling systems [54].

\section{Materials and methods}

\subsection{Two step anodizing and chemical etching}

Before anodizing of Aluminum samples (Merck KGaA, 99.95\%, $0.3 \mathrm{~mm}$ thickness - annealed), electrodes are electropolished $\left(\mathrm{A}=1 \mathrm{~cm}^{2}\right)$ in $\mathrm{HClO}_{4} 60$ wt. \% solution. In fact, the electropolishing voltage and temperature are fixed at $2 \mathrm{~V}$ and room temperature $\left(25^{\circ} \mathrm{C}\right)$ respectively. Also, the electropolishing time is optimized at $5 \mathrm{~min}$ in order to achieve a highly smooth surface without primary amorphous Aluminum Oxide. In both the first and second steps of anodization procedure, the electrodes are anodized in $\mathrm{C}_{2} \mathrm{H}_{2} \mathrm{O}_{4} 0.3 \mathrm{M}$ solution. Furthermore, anodization time, voltage, and temperature are fixed at $2 \mathrm{~h}, 40 \mathrm{~V}$ and room temperature $\left(25^{\circ} \mathrm{C}\right)$ respectively for first and second steps of this anodizing procedure. Fabricated porous Aluminum Oxides after the first step of anodization are etched in a solution of $0.6 \mathrm{M} \mathrm{H}_{3} \mathrm{PO}_{4} 85$ wt. $\%-0.2 \mathrm{M} \mathrm{H}_{2} \mathrm{CrO}_{4}$. The etching temperature and time are fixed at $60^{\circ} \mathrm{C}$ and $30 \mathrm{~min}$ respectively. Finally, the similar chemical etching procedure by using $5 \mathrm{wt}$. $\%$ phosphoric acid solution is done at room temperature for 40min in order to widen the pores of self-assembled template and convert it to Aluminum Oxide nanowires.

\subsection{Materials characterization}

Samples' characterization, which was used to investigate morphology of self-assembled templates as well as Aluminum Oxide nanowires, was done with a field emission scanning electron microscope (FE-SEM) Quanta 3D FEG (FEI, Phillips, The Netherlands). In order to examine the pore sizes as well as morphology (i.e. order structure) of nanowires, ImageJ [55] image processing software is used to obtain quantitative information on the average diameter of the self-assembled templates' pores' diameter and length, as well as diameter 
of the fabricated Aluminum Oxide nanowires. The crystalline structure of the Aluminum Oxide nanowires was analyzed by X-ray diffraction using a Rigaku Ultima IV diffractometer with Co $\mathrm{K}$ radiation and operating parameters of $40 \mathrm{~mA}$ and $40 \mathrm{kV}$ with a scanning speed of $1^{\circ}$ per minute and step size of $0.02^{\circ}$.

\section{Results and discussion}

\subsection{Self-assembled template morphology}

The porous morphology of two steps anodized Aluminum Oxide is shown in fig. 1. According to fig. 1(a), which is analyzed by using image processing techniques, it could be understood that the average pore size of this self-assembled template is $60 \mathrm{~nm}$. Furthermore, the order structure of this porous medium is analyzed by using the fast Fourier transform (FFT) technique in order to examine the spatial structure of pores and their deviation from honeycomb structure. As a result, according to fig. 1(b), the FFT result of this AAO microstructure shows 6 strong bright dots, which indicates that a perfect honeycomb structure is achieved after the second step of anodization. Additionally, in order to examine the aspect ratio of self-assembled pores of AAO, a cross-sectional FE-SEM microscopy is done to estimate the thickness of Aluminum Oxide after the second step of anodization (cref. fig. 2). As shown in fig. 2(a), the thickness of AAO template is about $33 \boldsymbol{\mu} \mathrm{m}$. As a result, the aspect ratio of pores, which is defined as the ratio of thickness over diameter, could be estimated as 550. Also, this aspect ratio will be increased for nanowires after AAO dissolution because of nanowires' radial shrinkage due to compressive residual stresses $[56,57]$. The wall thickness of pores in self-assembled AAO template is estimated as $50 \mathrm{~nm}$, which is shown in fig. 2(b). This highly ordered structure after the second step of anodization is achieved because: the quantum dots are created on the electrode's surface after etching step, which could facilitate the directional growth of AAO as well as controlling of its diameter more precisely [58].

\subsection{Aluminum Oxide nanowires}

FE-SEM microscopy technique is used to investigate the morphology of Aluminum Oxide nanowires after final chemical etching of AAO template. In fig. 3, Aluminum Oxide nanowires are shown in two different resolutions, which show their long-range order structure (cref. fig. 3(a)) as well as the diameter of the nanowires (cref. fig. 3(b), $67 \mathrm{~nm}$ ). As a result, according to fig. 3(b), it could be understood that the final diameter of Aluminum Oxide nanowires could be controlled by pore size and its wall thickness by changing the anodization voltage or chemical etching time. Also, the EDS and X-ray diffraction techniques are used to examine the chemical composition and crystalline structure of Aluminum Oxide nanowires respectively (cref. fig. 4). According to fig. 4(a), it shows that the synthesized Aluminum Oxide nanowires have a high purity and there is no residual chemical species from etching or anodizing solutions in the Aluminum Oxide chemical structure. Furthermore, fig. 4(b) shows that Aluminum Oxide nanowires have a preferential growth at (102) direction due to (102) maximum peak in their X-ray diffraction spectrum. This preferential growth observed in X-ray diffraction pattern of Aluminum Oxide nanowires indicates that crystalline structure of Aluminum Oxide based nanostructures could be optimized by 
changing anodization voltage as well as chemical composition of solution in the context of the proposed electrochemical method. Additionally, this oriented crystalline structure of Aluminum Oxide nanowires in (102) direction shows exceptional opteoelectronic properties for application in the micro-electronic devices due to their electronic confinement effects according to pore sizes of self-assembled templates which are comparable to high frequency electromagnetic spectra wavelengths (e.g. opteoelectronic band gap) [59-65].

\section{Conclusions}

In this research, an electrochemical technique is developed, which could be easily used to fabricate Aluminum Oxide nanowires with highly-ordered structure and reasonable controllability over the chemical composition as well as the crystalline structure. Additionally, this technique could facilitate the large-scale production of nanostructures due to its room temperature operating environment. As a result, this technique could be generalized to develop industrial scale coating facilities, which could be used in Lithium-ion battery production as well as other industries, such as: biomedical applications [66-70], oil and gas extraction plants [71-74], and nanoparticles technologies [75]. Finally, these Aluminum Oxide nanowires' production technique should be optimized by using the proposed electrochemical synthesizing procedure, and be examined in assembled micro-electronic devices to accurately measure their opteoelectronic properties. 


\section{References}

[1] A. Nazemi, A. Najafian, S. A. S. Sadjadi, Aluminium oxide nanowires synthesis from high purity aluminium films via two-step anodization, Superlattices and Microstructures 81 (2015) 1 - 6 . doi:https://doi.org/10.1016/j.spmi.2015.01.013.

URL http://www.sciencedirect.com/science/article/pii/S0749603615000348

[2] L. Meier, A. Alvarez, D. Salinas, M. del Barrio, Formation of dense alumina nanowires from anodic alumina membranes, Materials Letters 85 (2012) 146 - 148. doi:https://doi.org/10.1016/j.matlet.2012.06.085.

URL http://www.sciencedirect.com/science/article/pii/S0167577X12009329

[3] Z. Su, X. Li, F. Qu, H. Zhou, L. Liu, Q. Zhou, L. Zhang, H. Wang, M. Feng, Y. Wang, X. Cao, Investigation of the super-long alumina nanowire array synthesized with a novel method, Solid State Communications 149 (41) (2009) 1782 - 1785. doi:https://doi.org/10.1016/j.ssc.2009.07.020.

URL http://www.sciencedirect.com/science/article/pii/S0038109809004438

[4] K. Zhu, J. Cheng, M. Chang, W. Wang, W. Wei, G. Ge, Growth of alumina oxide nanowires in an aluminum anodization process, Chinese Science Bulletin 56 (18) (2011) 1947. doi:10.1007/s11434-011$4507-y$.

URL https://doi.org/10.1007/s11434-011-4507-y

[5] Z. L. Xiao, C. Y. Han, U. Welp, H. H. Wang, W. K. Kwok, G. A. Willing, J. M. Hiller, R. E. Cook, D. J. Miller, G. W. Crabtree, Fabrication of alumina nanotubes and nanowires by etching porous alumina membranes, Nano Letters 2 (11) (2002) 1293-1297. arXiv:https://doi.org/10.1021/nl025758q,

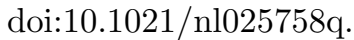

URL https://doi.org/10.1021/nl025758q

[6] G. Xiong, J. W. Elam, H. Feng, C. Y. Han, H.-H. Wang, L. E. Iton, L. A. Curtiss, M. J. Pellin, M. Kung, H. Kung, P. C. Stair, Effect of atomic layer deposition coatings on the surface structure of anodic aluminum oxide membranes, The Journal of Physical Chemistry B 109 (29) (2005) 14059-14063, pMID: 16852765. arXiv:https://doi.org/10.1021/jp0503415, doi:10.1021/jp0503415. URL https://doi.org/10.1021/jp0503415

[7] A. Alabi, A. AlHajaj, L. Cseri, G. Szekely, P. Budd, L. Zou, Review of nanomaterials-assisted ion exchange membranes for electromembrane desalination, npj Clean Water 1 (1) (2018) 10. doi:10.1038/s41545-018-0009-7. URL https://doi.org/10.1038/s41545-018-0009-7

[8] Z. Wang, A. Wu, L. Colombi Ciacchi, G. Wei, Recent advances in nanoporous membranes for water purification, Nanomaterials (Basel) 8 (2) (2018) 65, nanomaterials-08-00065[PII]. doi: $10.3390 /$ nano8020065.

URL http://www.ncbi.nlm.nih.gov/pmc/articles/PMC5853697/

[9] R. Li, L. Zhang, P. Wang, Rational design of nanomaterials for water treatment, Nanoscale 7 (2015) 17167-17194. doi:10.1039/C5NR04870B.

URL http://dx.doi.org/10.1039/C5NR04870B

[10] L.-H. Yang, Y.-J. Wang, Y.-L. Chueh, L.-J. Chen, 3d-nanorod fenton-like cuo/tio2 photocatalyst by electrodeposition in aao template for dye degradation, Meeting Abstracts MA2018-01 (40) (2018) 2327. URL http://ma.ecsdl.org/content/MA2018-01/40/2327. abstract

[11] J.-B. Shi, C.-J. Chen, Y.-T. Lin, W.-C. Hsu, Y.-C. Chen, P.-F. Wu, Anodic aluminum oxide membraneassisted fabrication of b-in(2)s(3)nanowires, Nanoscale Res Lett 4 (9) (2009) 1059-1063, 1556-276X-41059[PII]. doi:10.1007/s11671-009-9357-z.

URL http://www.ncbi.nlm.nih.gov/pmc/articles/PMC2894172/

[12] D. N. Kelly, R. H. Wakabayashi, A. M. Stacy, A modified solgel technique for pore size control in porous aluminum oxide nanowire templates, ACS Applied Materials \& Interfaces 6 (22) (2014) 20122-20129, pMID: 25425125. arXiv:https://doi.org/10.1021/am5056867, doi:10.1021/am5056867.

URL https://doi.org/10.1021/am5056867

[13] W. Lee, S.-J. Park, Porous anodic aluminum oxide: Anodization and templated synthesis of functional nanostructures, Chemical Reviews 114 (15) (2014) 7487-7556, pMID: 24926524. 
arXiv:https://doi.org/10.1021/cr500002z, doi:10.1021/cr500002z.

URL https://doi.org/10.1021/cr500002z

[14] D. Xu, G. Guo, Template Assisted Synthesis of Semiconductor Nanowires, Springer US, Boston, MA, 2003, pp. 317-341. doi:10.1007/978-0-387-28745-4_10.

URL https://doi.org/10.1007/978-0-387-28745-4_10

[15] P. Milka, I. Krest, M. Keusgen, , Biotechnology and Bioengineering 69 (3) 344-348.

[16] B. Reddy, B. R. Dorvel, J. Go, P. R. Nair, O. H. Elibol, G. M. Credo, J. S. Daniels, E. K. C. Chow, X. Su, M. Varma, M. A. Alam, R. Bashir, High-k dielectric al(2)o(3) nanowire and nanoplate field effect sensors for improved ph sensing, Biomed Microdevices 13 (2) (2011) 335-344, 21203849[pmid]. doi:10.1007/s10544-010-9497-z.

URL http://www.ncbi.nlm.nih.gov/pmc/articles/PMC3190593/

[17] B. Sun, Y. Sun, C. Wang, Encoding optoelectrical sub-components in an al2o3 nanowire for rewritable high-resolution nanopatterning, Nano Letters 18 (7) (2018) 4172-4179, pMID: 29893568. arXiv:https://doi.org/10.1021/acs.nanolett.8b00985, doi:10.1021/acs.nanolett.8b00985.

URL https://doi.org/10.1021/acs.nanolett.8b00985

[18] Z. Gu, F. Liu, J. Y. Howe, M. Parans Paranthaman, Z. Pan, Germanium-catalyzed hierarchical al2o3 and sio2nanowire bunch arrays, Nanoscale 1 (2009) 347-354. doi:10.1039/B9NR00040B. URL http://dx.doi.org/10.1039/B9NR00040B

[19] W. Zhou, X. Dai, T.-M. Fu, C. Xie, J. Liu, C. M. Lieber, Long term stability of nanowire nanoelectronics in physiological environments, Nano Letters 14 (3) (2014) 1614-1619, pMID: 24479700. arXiv:https://doi.org/10.1021/nl500070h, doi:10.1021/nl500070h. URL https://doi.org/10.1021/n1500070h

[20] X. Geng, Z. Jiao, H. Wang, Q. Fu, H. Zhong, Z. Li, The al2o3 nanowire grown on silicon chips by electrochemical reaction under afm probe, in: 2006 1st IEEE International Conference on Nano/Micro Engineered and Molecular Systems, 2006, pp. 679-681. doi:10.1109/NEMS.2006.334871.

[21] J.-Y. KIM, K. NOH, C. CHOI, K. S. BRAMMER, M. LOYA, L.-H. CHEN, S. JIN, Optically transparent glass with vertically aligned surface al2o3 nanowires having superhydrophobic characteristics, Nano 05 (02) (2010) 89-95. arXiv:https://doi.org/10.1142/S1793292010001962, doi:10.1142/S1793292010001962.

URL https://doi.org/10.1142/S1793292010001962

[22] J. K. Vohs, A. Bentz, K. Eleamos, J. Poole, B. D. Fahlman, Chemical vapor deposition of aluminum oxide thin films, Journal of Chemical Education 87 (10) (2010) 1102-1104. arXiv:https://doi.org/10.1021/ed100391p, doi:10.1021/ed100391p. URL https://doi.org/10.1021/ed100391p

[23] J. Benson, S. Boukhalfa, A. Magasinski, A. Kvit, G. Yushin, Chemical vapor deposition of aluminum nanowires on metal substrates for electrical energy storage applications, ACS Nano 6 (1) (2012) 118125, pMID: 22166004. arXiv:https://doi.org/10.1021/nn202979y, doi:10.1021/nn202979y. URL https://doi.org/10.1021/nn202979y

[24] A. K. Keshri, J. Huang, V. Singh, W. Choi, S. Seal, A. Agarwal, Synthesis of aluminum oxide coating with carbon nanotube reinforcement produced by chemical vapor deposition for improved fracture and wear resistance, Carbon 48 (2) (2010) 431 - 442. doi:https://doi.org/10.1016/j.carbon.2009.08.046. URL http://www.sciencedirect.com/science/article/pii/S0008622309006381

[25] Y. Zhao, C. Li, M. Chen, X. Yu, Y. Chang, A. Chen, H. Zhu, Z. Tang, Growth of aligned zno nanowires via modified atmospheric pressure chemical vapor deposition, Physics Letters A 380 (47) (2016) 3993 - 3997. doi:https://doi.org/10.1016/j.physleta.2016.06.030.

URL http://www.sciencedirect.com/science/article/pii/S0375960116303528

[26] B. H. Kim, J. W. Kwon, Metal catalyst for low-temperature growth of controlled zinc oxide nanowires on arbitrary substrates, Scientific Reports 4 (2014) 4379 EP -, article. URL http://dx.doi.org/10.1038/srep04379

[27] M. M. Tavakoli, A. Waleed, L. Gu, D. Zhang, R. Tavakoli, B. Lei, W. Su, F. Fang, Z. Fan, A noncatalytic vapor growth regime for organohalide perovskite nanowires using anodic aluminum oxide 
templates, Nanoscale 9 (2017) 5828-5834. doi:10.1039/C7NR00444C. URL http://dx.doi.org/10.1039/C7NR00444C

[28] J. jun LI, L. ting ZHANG, J. bo ZHU, Y. LIU, W. chang HAO, Controllable synthesis of zn0.95co0.05o nanowires and nanotubes by electrophoretic deposition method, Transactions of Nonferrous Metals Society of China 22 (2012) s95 - s99. doi:https://doi.org/10.1016/S1003-6326(12)61690-2. URL http://www.sciencedirect.com/science/article/pii/S1003632612616902

[29] X.-S. Fang, C.-H. Ye, X.-X. Xu, T. Xie, Y.-C. Wu, L.-D. Zhang, Synthesis and photoluminescence of -al 2 o 3 nanowires, Journal of Physics: Condensed Matter 16 (23) (2004) 4157.

URL http://stacks . iop. org/0953-8984/16/i=23/a=030

[30] G.-J. Wang, S.-W. Chou, Electrophoretic deposition of uniformly distributed tio 2 nanoparticles using an anodic aluminum oxide template for efficient photolysis, Nanotechnology 21 (11) (2010) 115206. URL http://stacks.iop.org/0957-4484/21/i=11/a=115206

[31] Y. Lin, Q. Lin, X. Liu, Y. Gao, J. He, W. Wang, Z. Fan, A highly controllable electrochemical anodization process to fabricate porous anodic aluminum oxide membranes, Nanoscale Res Lett 10 (2015) 495, 1202[PII]. doi:10.1186/s11671-015-1202-y.

URL http://www.ncbi.nlm.nih.gov/pmc/articles/PMC4691247/

[32] M. Lanki, A. Nourmohammadi, M. H. Feiz, Electrophoretic growth of pbtio3 nanotubes, Ferroelectrics 448 (1) (2013) 134-144. arXiv:https://doi.org/10.1080/00150193.2013.822740, doi:10.1080/00150193.2013.822740.

URL https://doi.org/10.1080/00150193.2013.822740

[33] Y. Shu-Min, G. Jian-Jun, W. Su-Xin, Q. Yun-Kai, Study on physical properties of anodic aluminum oxide films modified by co nanodots, Thin Solid Films 660 (2018) 82 - 87 . doi:https://doi.org/10.1016/j.tsf.2018.03.070.

URL http://www.sciencedirect.com/science/article/pii/S0040609018302207

[34] A. Maghsodi, L. Adlnasab, M. Shabanian, M. Javanbakht, Optimization of effective parameters in the synthesis of nanopore anodic aluminum oxide membrane and arsenic removal by prepared magnetic iron oxide nanoparicles in anodic aluminum oxide membrane via ultrasonic-hydrothermal method, Ultrasonics Sonochemistry 48 (2018) 441 - 452. doi:https://doi.org/10.1016/j.ultsonch.2018.07.003. URL http://www.sciencedirect.com/science/article/pii/S135041771830395X

[35] F.-Y. Wen, P.-S. Chen, T.-W. Liao, Y.-J. Juang, Microwell-assisted filtration with anodic aluminum oxide membrane for raman analysis of algal cells, Algal Research 33 (2018) 412 - 418. doi:https://doi.org/10.1016/j.algal.2018.06.022.

URL http://www.sciencedirect.com/science/article/pii/S2211926417310342

[36] C.-W. Hun, C.-C. Chang, S.-H. Chen, C. C. Chen, A. Fang, Y.-L. Kuo, Transparent sapphire substrates with tunable optical properties by decorating with nanometric oxide on porous anodic aluminum oxide patterns, Ceramics International 44 (9) (2018) 10898 - 10906. doi:https://doi.org/10.1016/j.ceramint.2018.03.145.

URL http://www.sciencedirect.com/science/article/pii/S0272884218307119

[37] U. K. Chime, F. I. Ezema, J. Marques-Hueso, Porosity and hole diameter tuning on nanoporous anodic aluminium oxide membranes by one-step anodization, Optik 174 (2018) 558 - 562 . doi:https://doi.org/10.1016/j.ijleo.2018.08.109.

URL http://www.sciencedirect.com/science/article/pii/S0030402618312592

[38] S. Dadras, M. Faraji, Improved carbon nanotube growth inside an anodic aluminum oxide template using microwave radiation, Journal of Physics and Chemistry of Solids 116 (2018) $203-208$. doi:https://doi.org/10.1016/j.jpcs.2018.01.039.

URL http://www.sciencedirect.com/science/article/pii/S002236971731380X

[39] L. Winter, K. Hockauf, T. Lampke, High cycle fatigue behavior of the severely plastically deformed 6082 aluminum alloy with an anodic and plasma electrolytic oxide coating, Surface and Coatings Technology 349 (2018) 576 - 583. doi:https://doi.org/10.1016/j.surfcoat.2018.06.044.

URL http://www.sciencedirect.com/science/article/pii/S0257897218306194

[40] M. Hakamada, N. Miyazawa, Y. Kohashi, Y. Yamano, M. Mabuchi, Nano-anchor effect by 
anodic oxidation of aluminum sheets in joining by electrodeposition, Procedia Manufacturing 15 (2018) 1416 - 1421, proceedings of the 17th International Conference on Metal Forming METAL FORMING 2018 September 16 19, 2018, Loisir Hotel Toyohashi, Toyohashi, Japan. doi:https://doi.org/10.1016/j.promfg.2018.07.340.

URL http://www.sciencedirect.com/science/article/pii/S2351978918310369

[41] D. Peng, J. Chen, L. Jiao, Y. Liu, A fast-responding semi-transparent pressure-sensitive paint based on through-hole anodized aluminum oxide membrane, Sensors and Actuators A: Physical 274 (2018) 10 - 18. doi:https://doi.org/10.1016/j.sna.2018.02.026.

URL http://www.sciencedirect.com/science/article/pii/S0924424717321581

[42] L. Pan, A. Zhang, Z. Zheng, L. Duan, L. Zhang, Y. Shi, J. Tao, Enhancing interfacial strength between aa5083 and cryogenic adhesive via anodic oxidation and silanization, International Journal of Adhesion and Adhesives 84 (2018) 317 - 324. doi:https://doi.org/10.1016/j.ijadhadh.2018.04.006.

URL http://www.sciencedirect.com/science/article/pii/S0143749618300988

[43] R. Chaisen, W. Nhuapeng, W. Thamjaree, Coating of molybdenum oxide on anodized aluminium plate applied for ultracapacitor electrodes, Materials Today: Proceedings 5 (5, Part 1) (2018) 10979 - 10983, the 5th Thailand International Nanotechnology Conference (NanoThailand2016), November 27-29, 2016. doi:https://doi.org/10.1016/j.matpr.2018.01.012.

URL http://www.sciencedirect.com/science/article/pii/S221478531830018X

[44] J. Yang, S. Qu, H. Ma, J. Wang, S. Yang, Y. Pang, Broadband infrared metamaterial absorber based on anodic aluminum oxide template, Optics Laser Technology 101 (2018) 177 - 182. doi:https://doi.org/10.1016/j.optlastec.2017.10.032.

URL http://www.sciencedirect.com/science/article/pii/S0030399217310873

[45] S. Ate, E. Baran, B. Yazc, The nanoporous anodic alumina oxide formed by two-step anodization, Thin Solid Films 648 (2018) 94 - 102. doi:https://doi.org/10.1016/j.tsf.2018.01.013.

URL http://www.sciencedirect.com/science/article/pii/S004060901830021X

[46] L. Sacco, I. Florea, M. Chtelet, C.-S. Cojocaru, Investigation of porous anodic alumina templates formed by anodization of single-crystal aluminum substrates, Thin Solid Films 660 (2018) 213 - 220. doi:https://doi.org/10.1016/j.tsf.2018.06.015.

URL http://www.sciencedirect.com/science/article/pii/S0040609018304097

[47] J. Lu, G. Wei, Y. Yu, C. Guo, L. Jiang, Aluminum alloy aa2024 anodized from the mixed acid system with enhanced mechanical properties, Surfaces and Interfaces 13 (2018) 46 - 50 . doi:https://doi.org/10.1016/j.surfin.2018.08.003.

URL http://www.sciencedirect.com/science/article/pii/S2468023018300300

[48] W. Tao, D. Pan, Z. Gong, X. Peng, Nanoporous platinum electrode grown on anodic aluminum oxide membrane: Fabrication, characterization, electrocatalytic activity toward reactive oxygen and nitrogen species, Analytica Chimica Actadoi:https://doi.org/10.1016/j.aca.2018.06.076.

URL http://www.sciencedirect.com/science/article/pii/S0003267018308419

[49] M. Michalska-Domaska, W. J. Stpniowski, M. Salerno, Effect of inter-electrode separation in the fabrication of nanoporous alumina by anodization, Journal of Electroanalytical Chemistry 823 (2018) 47 - 53. doi:https://doi.org/10.1016/j.jelechem.2018.05.038.

URL http://www.sciencedirect.com/science/article/pii/S1572665718303928

[50] T. Xue, Q. Xu, Y.-X. Li, H.-Y. Qi, Z.-B. Wang, Y. Yang, T.-L. Ren, A method of controlling the hole size of nanopores array on anodic aluminum oxide, Materials Letters 215 (2018) 183 - 186. doi:https://doi.org/10.1016/j.matlet.2017.12.042.

URL http://www.sciencedirect.com/science/article/pii/S0167577X17318013

[51] S. A. Abdel-Gawad, W. M. Osman, A. M. Fekry, Characterization and corrosion behavior of anodized aluminum alloys for military industries applications in artificial seawater, Surfaces and Interfacesdoi:https://doi.org/10.1016/j.surfin.2018.08.001.

URL http://www.sciencedirect.com/science/article/pii/S2468023018300439

[52] M. R. Pallaka, D. K. Unruh, S. L. Simon, Melting behavior of n-alkanes in anodic aluminum oxide (aao) nanopores using flash differential scanning calorimetry, Thermochimica Acta 663 (2018) 157 - 
164. doi:https://doi.org/10.1016/j.tca.2018.01.016.

URL http://www.sciencedirect.com/science/article/pii/S004060311830025X

[53] G. A. Gelves, Z. T. M. Murakami, M. J. Krantz, J. A. Haber, Multigram synthesis of copper nanowires using ac electrodeposition into porous aluminium oxide templates, J. Mater. Chem. 16 (2006) 30753083. doi:10.1039/B603442J.

URL http://dx.doi.org/10.1039/B603442J

[54] P. G. Schiavi, P. Altimari, A. Rubino, F. Pagnanelli, Electrodeposition of cobalt nanowires into alumina templates generated by one-step anodization, Electrochimica Acta 259 (2018) 711 - 722 . doi:https://doi.org/10.1016/j.electacta.2017.11.035.

URL http://www.sciencedirect.com/science/article/pii/S0013468617323861

[55] C. T. Rueden, J. Schindelin, M. C. Hiner, B. E. DeZonia, A. E. Walter, E. T. Arena, K. W. Eliceiri, Imagej2: Imagej for the next generation of scientific image data, BMC Bioinformatics 18 (1) (2017) 529. doi:10.1186/s12859-017-1934-z.

URL https://doi.org/10.1186/s12859-017-1934-z

[56] N. Tasaltin, S. Öztürk, N. Kilinç, H. Yüzer, Z. Öztürk, Fabrication of vertically aligned pd nanowire array in aao template by electrodeposition using neutral electrolyte, Nanoscale Res Lett 5 (7) (2010) 1137-1143, 1556-276X-5-1137[PII]. doi:10.1007/s11671-010-9616-z.

URL http://www.ncbi.nlm.nih.gov/pmc/articles/PMC2894036/

[57] T. Gorisse, L. Dupré, P. Gentile, M. Martin, M. Zelsmann, D. Buttard, Highly organised and dense

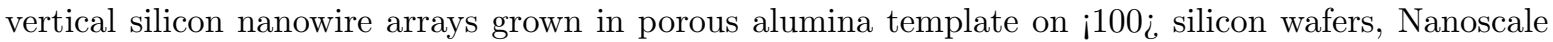
Research Letters 8 (1) (2013) 287. doi:10.1186/1556-276X-8-287.

URL https://doi.org/10.1186/1556-276X-8-287

[58] J. Liang, H. Luo, R. Beresford, J. Xu, A growth pathway for highly ordered quantum dot arrays, Applied Physics Letters 85 (24) (2004) 5974-5976. arXiv:https://doi.org/10.1063/1.1834987, doi:10.1063/1.1834987.

URL https://doi.org/10.1063/1.1834987

[59] M.-L. Lu, T.-M. Weng, J.-Y. Chen, Y.-F. Chen, Ultrahigh-gain single sno2 nanowire photodetectors made with ferromagnetic nickel electrodes, Npg Asia Materials 4 (2012) e26 EP -, original Article. URL http://dx.doi.org/10.1038/am.2012.48

[60] P. Shimpi, P.-X. Gao, D. G. Goberman, Y. Ding, Low temperature synthesis and characterization of mgo/zno composite nanowire arrays, Nanotechnology 20 (12) (2009) 125608.

URL http://stacks.iop.org/0957-4484/20/i=12/a=125608

[61] E. O. Filatova, A. S. Konashuk, Interpretation of the changing the band gap of al2o3 depending on its crystalline form: Connection with different local symmetries, The Journal of Physical Chemistry C 119 (35) (2015) 20755-20761. arXiv:https://doi.org/10.1021/acs.jpcc.5b06843, doi:10.1021/acs.jpcc.5b06843.

URL https://doi.org/10.1021/acs.jpcc.5b06843

[62] J. Gangwar, B. K. Gupta, P. Kumar, S. K. Tripathi, A. K. Srivastava, Time-resolved and photoluminescence spectroscopy of -al2o3 nanowires for promising fast optical sensor applications, Dalton Trans. 43 (2014) 17034-17043. doi:10.1039/C4DT01831A.

URL http://dx.doi.org/10.1039/C4DT01831A

[63] P. Prashanth, R. Raveendra, R. H. Krishna, S. Ananda, N. Bhagya, B. Nagabhushana, K. Lingaraju, H. R. Naika, Synthesis, characterizations, antibacterial and photoluminescence studies of solution combustion-derived -al2o3 nanoparticles, Journal of Asian Ceramic Societies 3 (3) (2015) 345 - 351. doi:https://doi.org/10.1016/j.jascer.2015.07.001.

URL http://www.sciencedirect.com/science/article/pii/S2187076415000639

[64] J. A. Alexander-Webber, C. K. Groschner, A. A. Sagade, G. Tainter, M. F. Gonzalez-Zalba, R. Di Pietro, J. Wong-Leung, H. H. Tan, C. Jagadish, S. Hofmann, H. J. Joyce, Engineering the photoresponse of inas nanowires, ACS Applied Materials \& Interfaces 9 (50) (2017) 43993-44000, pMID: 29171260. arXiv:https://doi.org/10.1021/acsami.7b14415, doi:10.1021/acsami.7b14415.

URL https://doi.org/10.1021/acsami.7b14415 
[65] C. Hahn, Z. Zhang, A. Fu, C. H. Wu, Y. J. Hwang, D. J. Gargas, P. Yang, Epitaxial growth of ingan nanowire arrays for light emitting diodes, ACS Nano 5 (5) (2011) 3970-3976, pMID: 21495684. arXiv:https://doi.org/10.1021/nn200521r, doi:10.1021/nn200521r.

URL https://doi.org/10.1021/nn200521r

[66] A. Amirjani, M. Yousefi, M. Cheshmaroo, Parametrical optimization of stent design; a numerical-based approach, Computational Materials Science $90 \quad(2014) \quad 210 \quad-\quad 220$. doi:https://doi.org/10.1016/j.commatsci.2014.04.002.

URL http://www.sciencedirect.com/science/article/pii/S092702561400233X

[67] N. W. Bressloff, G. Ragkousis, N. Curzen, Design optimisation of coronary artery stent systems, Annals of Biomedical Engineering 44 (2) (2016) 357-367. doi:10.1007/s10439-015-1373-9. URL https://doi.org/10.1007/s10439-015-1373-9

[68] Y.-H. Lim, H.-Y. Jeong, Finite element analyses for improved design of peripheral stents, Computer Methods in Biomechanics and Biomedical Engineering 20 (6) (2017) 653-662, pMID: 28349767. arXiv:https://doi.org/10.1080/10255842.2017.1286650, doi:10.1080/10255842.2017.1286650.

URL https://doi.org/10.1080/10255842.2017.1286650

[69] M. Basiaga, W. Walke, M. Antonowicz, A. Sambok-Kiebowicz, D. Nakonieczny, M. Gawlikowski, B. Zawidlak-Wgrzyska, C. Krawczyk, Effect of thin sio2 layers deposited by means of atomic layer deposition method on the mechanical and physical properties of stainless steel, Materialwissenschaft und Werkstofftechnik 49 (5) 562-567. arXiv:https://onlinelibrary.wiley.com/doi/pdf/10.1002/mawe.201700241, doi:10.1002/mawe.201700241.

URL https://onlinelibrary.wiley.com/doi/abs/10.1002/mawe. 201700241

[70] T. Sun, M. Yousefi, O. Oyekoya, C. U. Clemson, U. D. Schiller, 3 d visualization brain blood behavior , in vivo meets virtual reality, 2017.

[71] M. Yousefi, M. H. Farghadin, A. Farzadi, Investigate the causes of cracks in welded 310 stainless steel used in the flare tip, Engineering Failure Analysis 53 (2015) 138 - 147. doi:https://doi.org/10.1016/j.engfailanal.2015.04.002.

URL http://www.sciencedirect.com/science/article/pii/S1350630715001156

[72] M. Ghalambaz, M. Abdollahi, A. Eslami, A. Bahrami, A case study on failure of aisi 347h stabilized stainless steel pipe in a petrochemical plant, Case Studies in Engineering Failure Analysis 9 (2017) 52 - 62. doi:https://doi.org/10.1016/j.csefa.2017.07.001.

URL http://www.sciencedirect.com/science/article/pii/S2213290217300391

[73] I. Taie, A. Al-Shahrani, N. Qari, A. Fihri, W. Al-Obaid, G. Alabedi, High temperature corrosion resistant coatings for gas flare systems, Ceramics International 44 (5) (2018) $5124-5130$. doi:https://doi.org/10.1016/j.ceramint.2017.12.114.

URL http://www.sciencedirect.com/science/article/pii/S0272884217328201

[74] M. Yousefi, S. M. Musavi Khoei, Retained austenite measurement and carbide characterization study in cold work steels by 3d metallography method, Moldova, Republic of, 2012, p. 320. URL http://inis.iaea.org/search/search.aspx?orig_q=RN: 43124002

[75] M. Yousefi, M. M. Khoie, Molecular dynamics simulation of ni/cu-ni nanoparticles sintering under various crystallographic, thermodynamic and multi-nanoparticles conditions, The European Physical Journal D 69 (3) (2015) 71. doi:10.1140/epjd/e2015-50830-4.

URL https://doi.org/10.1140/epjd/e2015-50830-4 


\section{List of Figures}

1 Pore size distribution and FFT result of AAO template after two step anodization procedure. . . . . . . . . . . . . . . . 12

2 Thickness and pore wall estimation of AAO template, which could be used to calculate the aspect ratio of self-assembled pores. . . . . . . . . . . . . . 13

3 FE-SEM microscopy images of Aluminum Oxide nanowires after AAO template chemical etching. . . . . . . . . . . . . . . . . . . 14

4 EDS and X-ray diffraction spectra of Aluminum Oxide nanowires. . . . . . . 15 


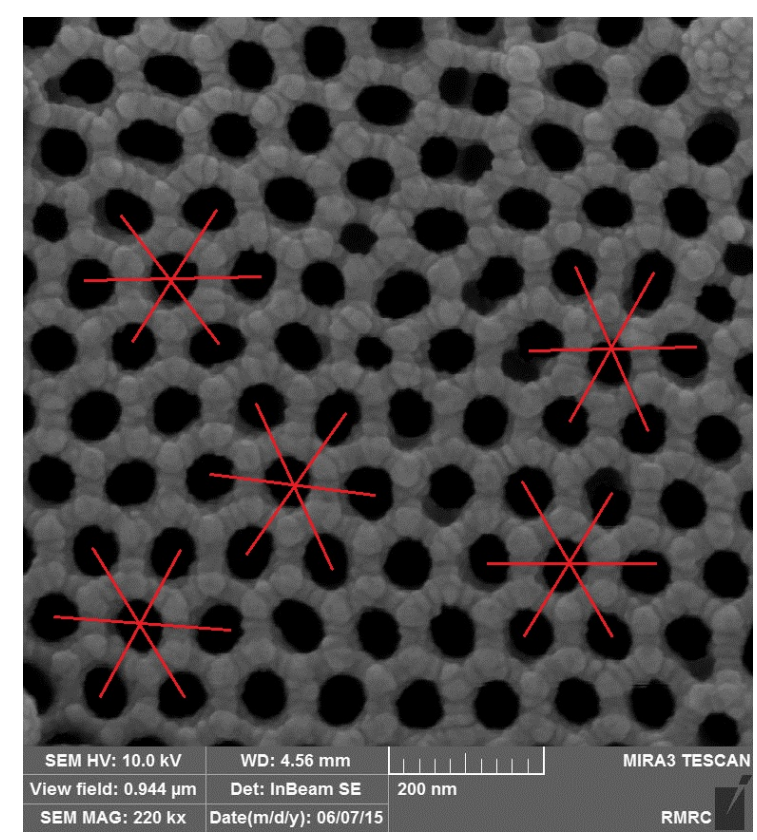

(a) Pore size distribution of AAO template.

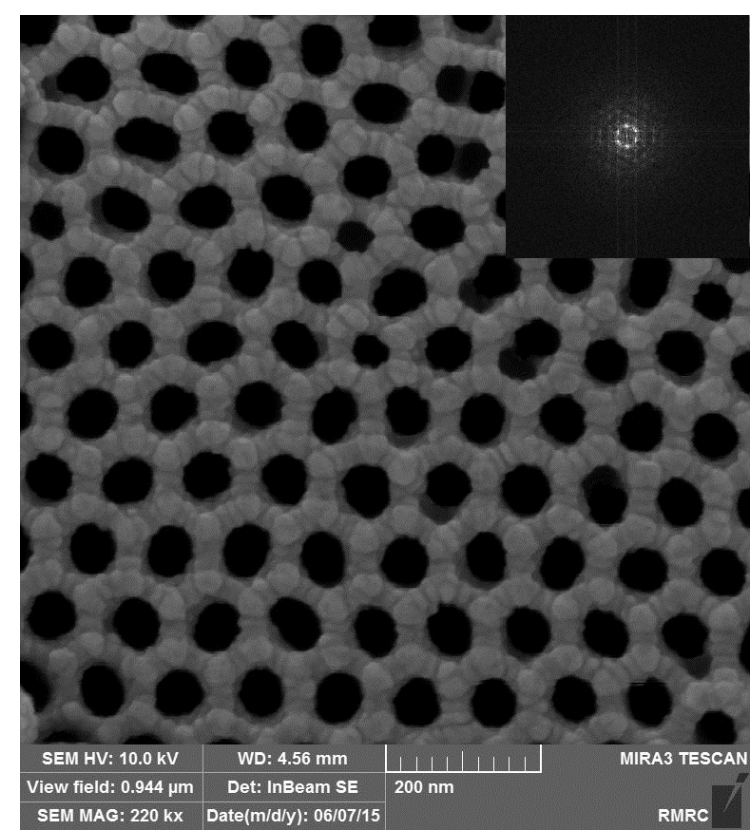

(b) FFT result of AAO template, which shows perfect honeycomb structure due to 6 bright dots in its FFT spectrum.

Figure 1: Pore size distribution and FFT result of AAO template after two step anodization procedure. 

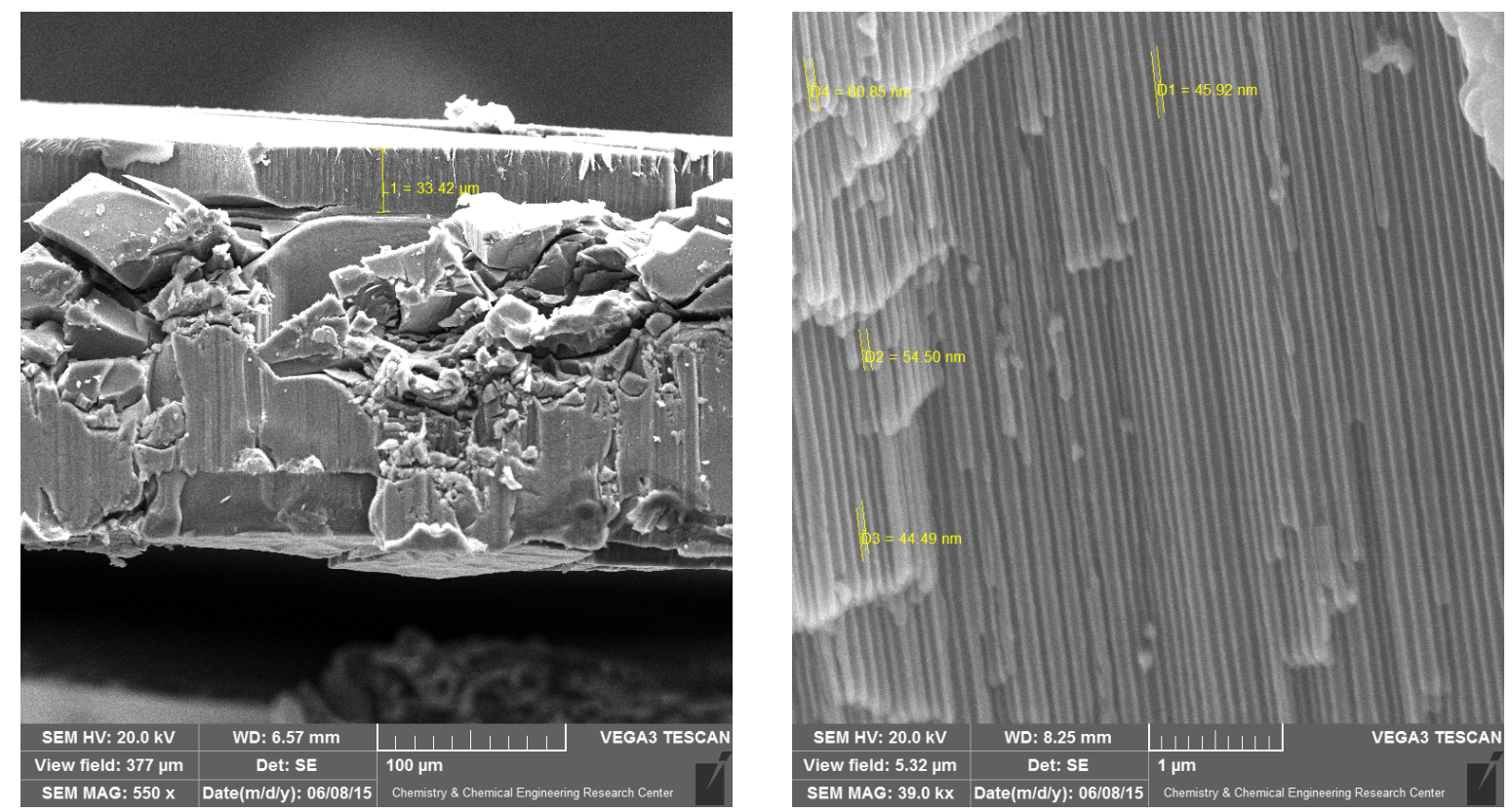

(a) Thickness estimation of AAO template.

(b) Pore wall estimation of AAO template.

Figure 2: Thickness and pore wall estimation of AAO template, which could be used to calculate the aspect ratio of self-assembled pores. 


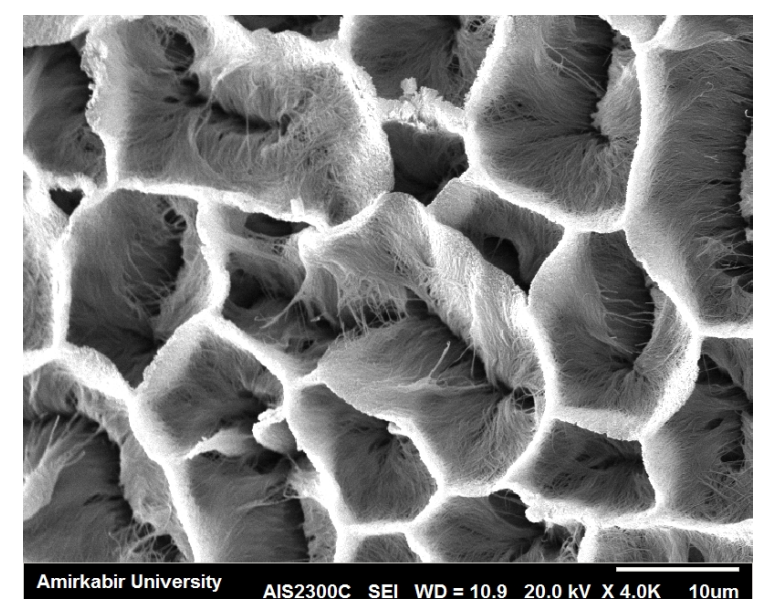

(a) Long-range order structure of Aluminum Oxide nanowires after AAO template chemical etching.

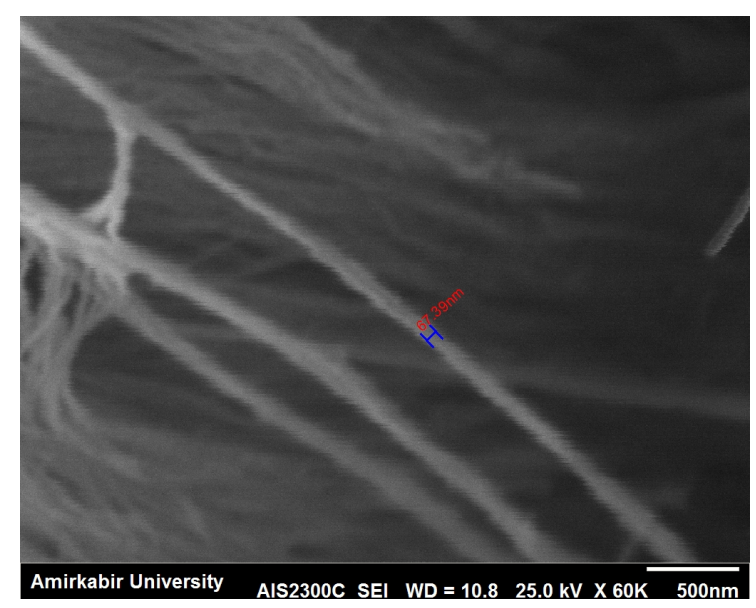

(b) Diameter estimation of Aluminum Oxide nanowires in order to estimate their aspect ratio.

Figure 3: FE-SEM microscopy images of Aluminum Oxide nanowires after AAO template chemical etching. 


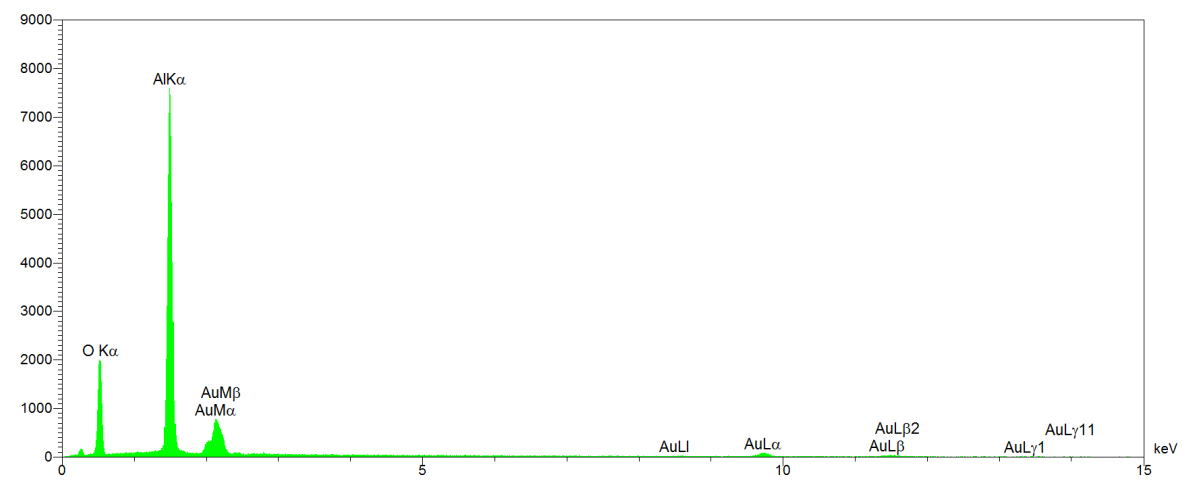

(a) EDS spectrum of Aluminum Oxide nanowires, which shows their chemical composition.

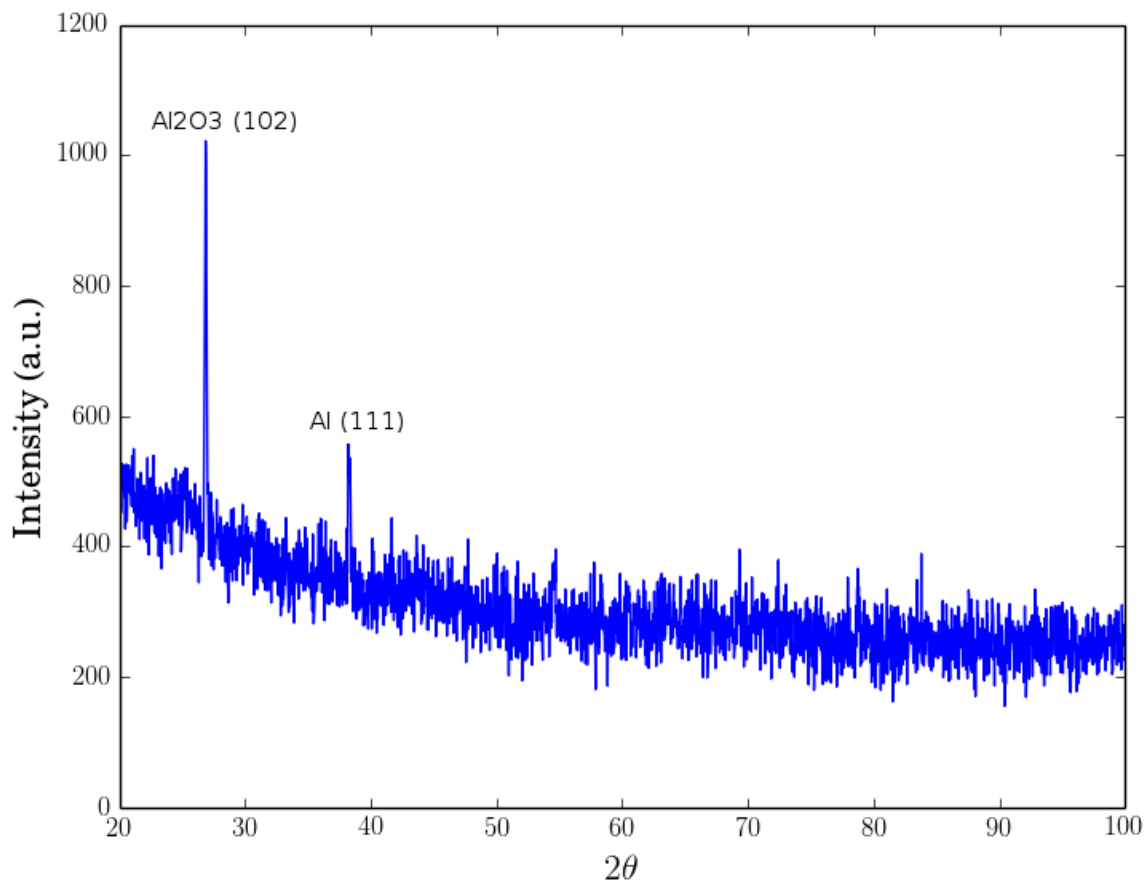

(b) X-ray diffraction spectrum of Aluminum Oxide nanowires as well as their crystalline structures.

Figure 4: EDS and X-ray diffraction spectra of Aluminum Oxide nanowires. 